\title{
The Multiple Continuum Components in the White Light Flare of 16 January 2009 on the dM4.5e Star YZ CMi
}

\author{
A.F. Kowalski ${ }^{1}$ - S.L. Hawley ${ }^{1}$. \\ J.A. Holtzman ${ }^{2}$. J.P. Wisniewski ${ }^{1,3}$. \\ E.J. Hilton ${ }^{1}$
}

(C) Springer $\bullet \bullet \bullet \bullet$

\begin{abstract}
The white light during $\mathrm{M}$ dwarf flares has long been known to exhibit the broadband shape of a $T \approx 10000 \mathrm{~K}$ blackbody, and the white light in solar flares is thought to arise primarily from Hydrogen recombination. Yet, a current lack of broad wavelength coverage solar-flare spectra in the optical/near-UV prohibits a direct comparison of the continuum properties to determine if they are indeed so different. New spectroscopic observations of a secondary flare during the decay of a megaflare on the dM4.5e star YZ CMi have revealed multiple components in the white-light continuum of stellar flares, including both a blackbody-like spectrum and a hydrogen recombination spectrum. One of the most surprising findings is that these two components are anti-correlated in their temporal evolution. We combine initial phenomenological modeling of the continuum components with spectra from radiative-hydrodynamic models to show that continuum veiling gives rise to the measured anti-correlation. This modeling allows us to use the components' inferred properties to predict how a similar spatially resolved, multiple-component white-light continuum might appear using analogies to several solar flare phenomena. We also compare the properties of the optical stellar flare white light to Ellerman bombs on the Sun.
\end{abstract}

Keywords: white light flares, solar-stellar connection, radiative transfer, Ellerman bombs

\section{Introduction}

In both solar and stellar flares, the near-UV and optical (white-light) continuum emission is an energetically important but unexplained phenomenon. On the Sun,

1 Astronomy Department, University of Washington, Box 351580, Seattle, WA 98195, USA. email: adamfk@u.washington.edu

${ }^{2}$ Department of Astronomy, New Mexico State University, Box 30001, Las Cruces, NM 88003, USA

${ }^{3}$ NSF Astronomy \& Astrophysics Postdoctoral Fellow. 
the white-light continuum appears in small regions of transient emission that are spatially and temporally coincident with hard X-ray bursts (Rust and Hegwer, 1975; Hudson et al., 1992; Neidig and Kane, 1993; Fletcher et al., 2007). This relation suggests that the origin of the white light is related to the energy deposited in the lower atmosphere by nonthermal electrons accelerated during flares. Broad wavelength coverage spectral observations are sparse and date back to several large solar flares from the 1970s and 1980s (Machado and Rust, 1974; Hiei, 1982; Neidig, 1983; Donati-Falchi. Smaldone, and Falciani, 1984). These spectra are consistent with continua arising primarily from the hydrogen Balmer continuum and $\mathrm{H}^{-}$emission.

Whereas the largest solar flares emit $<10^{32}$ ergs in the white-light continuum and last not much longer than ten minutes (e.g., Neidig. Grosser, and Hrovat, 1994), the white-light emission on active lower-mass M dwarfs can reach $>10^{34}$ ergs and persist for hours (Hawley and Pettersen, 1991; Kowalski et al., 2010a, hereafter K10a). More is known about the spectral shape of the white light during M dwarf flares (many spectrographs can easily obtain low-resolution, broad wavelength spectra of stellar flares, but it is difficult to place a spectrograph slit over solar white-light kernels, which are intermittent and largely unpredictable), which have been studied using broadband colors (Hawlev et al., 2003; Zhilvaev et al., 2007) and optical/NUV spectra (Hawlev and Pettersen, 1991; Eason et al., 1992; García-Alvarez et al., 2002; Fuhrmeister et al., 2008, K10a). In contrast to solar observations, the spectral shape during M dwarf flares suggests a hot blackbody with temperatures between $\approx 8500-11000$ $\mathrm{K}$. Areal coverages of this component are typically $<0.1 \%$ of the visible stellar hemisphere, which implies a compact geometry like those observed in white light at the footpoints of flare arcades on the Sun. Although this blackbody component seems to be nearly ubiquitous during (large) stellar flares, it is not predicted even by the most recent $1 \mathrm{D}$ radiative hydrodynamic (RHD) flare models Allred et al., 2006) produced with the RADYN code (Carlsson and Stein, 1994, 1995, 1997).

The "megaflare" of UT 16 January 2009 on the dM4.5e star YZ CMi is one of the longest lasting and most energetic flares observed on a low-mass single star. Low-resolution spectra $(3350-9260 \AA)$ were obtained in the flare's decay phase, which was elevated between 15 and 37 times the quiescent level and contained many secondary peaks. More than 160 spectra were obtained over 1.3 hours, and simultaneous U-band photometric observations of the entire flare event were provided by the NMSU $1 \mathrm{~m}$ telescope. A detailed description of the observations and data reduction is given in K10a.

In K10a, two continuum components were necessary to fit the blue (3350$5500 \AA)$ spectra: a hydrogen Balmer continuum $(\mathrm{BaC})$ component as predicted by the RHD models of Allred et al. $(2006)$ and a $T \approx 10000 \mathrm{~K}$ blackbody component. An intriguing anti-correlation was found between the temporal evolution of these two components: the blackbody emission increased when the $\mathrm{BaC}$ decreased, and vice versa (see Figure 1d of K10a). In this article, we revisit this anti-correlation and provide an explanation for it using the phenomenological models of the secondary flare spectra from Kowalski et al. 2010b, hereafter K10b). Finally, we show how each component of this flare might appear in the context of a solar flare "arcade". 


\section{Anti-Correlated Continuum Components and Continuum Veiling}

The anti-correlation between the blackbody and BaC components (K10a) can be understood qualitatively using Figure 1, where we show the spectral evolution of the total "flare-only" flux (denoted here as $F_{\lambda}^{\prime}$ ) during the rise phase of the secondary flare at $t \approx 130$ minutes. The spectra are color-coded to the nearest (in time) U-band measurement in the inset panel. At times prior to and near the beginning of the secondary flare (black, purple, and dark blue spectra), two distinct continuum components are clearly present in the spectra. The best-fit blackbody (short-dashed line) accounts for most continuum emission at $\lambda>$ $4000 \AA$, whereas the $\mathrm{BaC}$ emission above the blackbody is conspicuous at $\lambda<$ $3750 \AA$. During the rise and at the peak of the secondary flare (green, yellow, and red spectra), the $\mathrm{BaC}$ component seemingly disappears and the best-fit blackbody (long-dashed line) can fit the continuum shape throughout the entire wavelength range. K10a showed that the $\mathrm{H} \gamma$ line flux exhibits an anti-correlated relation with the blackbody component. This effect is also present in Figure 1 In the red (secondary flare peak) spectrum, the continuum at $\lambda \approx 4200 \AA$ is highest, yet the peaks of the hydrogen Balmer lines are lowest.

The secondary flares at $t \approx 95$ minutes and $t \approx 130$ minutes are events during which the blackbody flux becomes stronger while the $\mathrm{BaC}$ flux becomes weaker. K10a quantified this as an increasing filling factor (areal coverage; percent of stellar disk) of the blackbody (with constant temperature, $T=10000 \mathrm{~K}$ ) during the rise phase of each secondary flare. In Figure 1, we present an alternative interpretation. The blackbody curves (dashed lines) were fit to the spectra by allowing both the temperature and filling factor to vary. The best-fit blackbody temperatures and filling factors are $T \approx 10400 \mathrm{~K}$ and $X_{\mathrm{BB}} \approx 0.1 \%$ (at $t=122.9$ minutes; short-dashed line) and $T \approx 13000 \mathrm{~K}$ and $X_{\mathrm{BB}} \approx 0.1 \%$ (at $t=130$ minutes; long-dashed line). Strikingly, if both parameters are allowed to vary when fitting a blackbody function to these total flare spectra, the temperature increases by $\approx 2500 \mathrm{~K}$ while the filling factor remains approximately constant. Fitting a blackbody to the total flare spectrum (either by holding $T$ constant, or by allowing $X$ and $T$ to vary) gives only the average properties of the entire flaring region at that time. We next show that these interpretations can be improved by isolating the newly-formed flare emission.

K10b found that the isolated flare emission (denoted here as $F_{\lambda}^{\prime \prime}$ ) during the secondary flare's rise phase resembles the spectrum of a hot star, with the defining features being the hydrogen Balmer continuum and lines in absorption and a steeply rising continuum towards the blue at $\lambda>4000 \AA$ (Figure $1 \mathrm{~b}$ of K10b shows that the new flare emission, obtained by subtracting the pre-secondary flare spectrum (average of three black and purple spectra around $t=123.4$ minutes) from the average of two green-colored spectra around $t=126.5$ minutes in Figure 1 of this article, is very similar to the spectrum of the A0 star Vega). The observed anti-correlation between the continuum components in Figure 1 is a result of a 'hot star spectrum' forming during the secondary flare. The hot star ("blackbody-like") spectrum causes an increase in the continuum at $\lambda \approx 4200 \AA$ by an amount, $F_{4200}^{\prime \prime}$, whereas an increase in the continuum at $\lambda \approx 3500 \AA$ occurs by only $\approx 0.6 \times F_{4200}^{\prime \prime}$. In other words, the flux in the continuum on both sides of 
the Balmer jump increases, but the continuum at $\lambda \approx 4200 \AA$ increases by a larger amount. Thus, the apparent decrease in the total amount of $\mathrm{BaC}$ in emission from $t=123$ minutes to 130 minutes occurs as a result of 'continuum veiling' (similar to the continuum veiling observed for accreting T Tauri stars - see, e.g., Hartigan et al. (1989); Hessman and Guenther (1997); Herczeg and Hillenbrand (2008)).

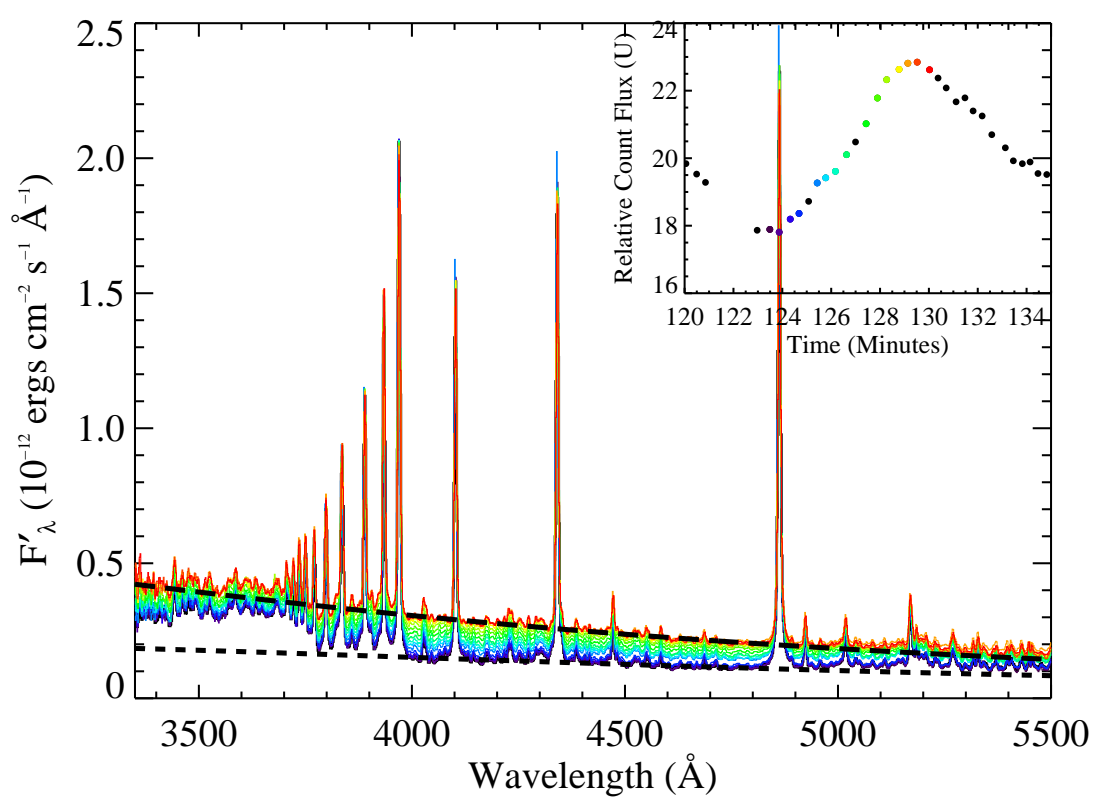

Figure 1. A series of 16 flare spectra obtained prior to the onset and through the peak of a secondary flare. The quiescent spectrum from 24 November 2008 has been subtracted, as in K10a. The U-band light curve (inset) is color-coded to the spectrum obtained closest in time. The best-fit blackbody curves to the black- and red-colored spectra are shown as the short-dashed $(T \approx 10400 \mathrm{~K})$ and long-dashed lines $(T \approx 13000 \mathrm{~K})$, respectively.

\section{Combining Continuum Components using Phenomenological Hot Spot Models}

In K10b, the $F_{\lambda}^{\prime \prime}$ emission was modelled phenomenologically with the static radiative transfer code, $\mathrm{RH}$ (Uitenbroek, 2001), as a temperature bump ("hot spot") with peak temperature $T=20000 \mathrm{~K}$, placed near the photosphere (below the temperature minimum) of the quiescent $\mathrm{M}$ dwarf atmosphere. Here, we use a sum of individual hot spots and the RHD model spectrum (hereafter RHDF11) of Allred et al. (2006) to model the total flare emission $\left(F_{\lambda}^{\prime}\right)$ at two times during the megaflare on YZ CMi. Figure 2 shows flare spectra from Figure 1 averaged around $t=123.4$ minutes ( $F 0^{\prime}$; grey) and at $t=126.5$ minutes $\left(F 1^{\prime}\right.$; black). 
These are the spectra corresponding to times immediately before and nearly half-way up the rise phase of the secondary flare, respectively (i.e., the same two spectra presented in Figure 1a of K10b but with the quiescent level subtracted).

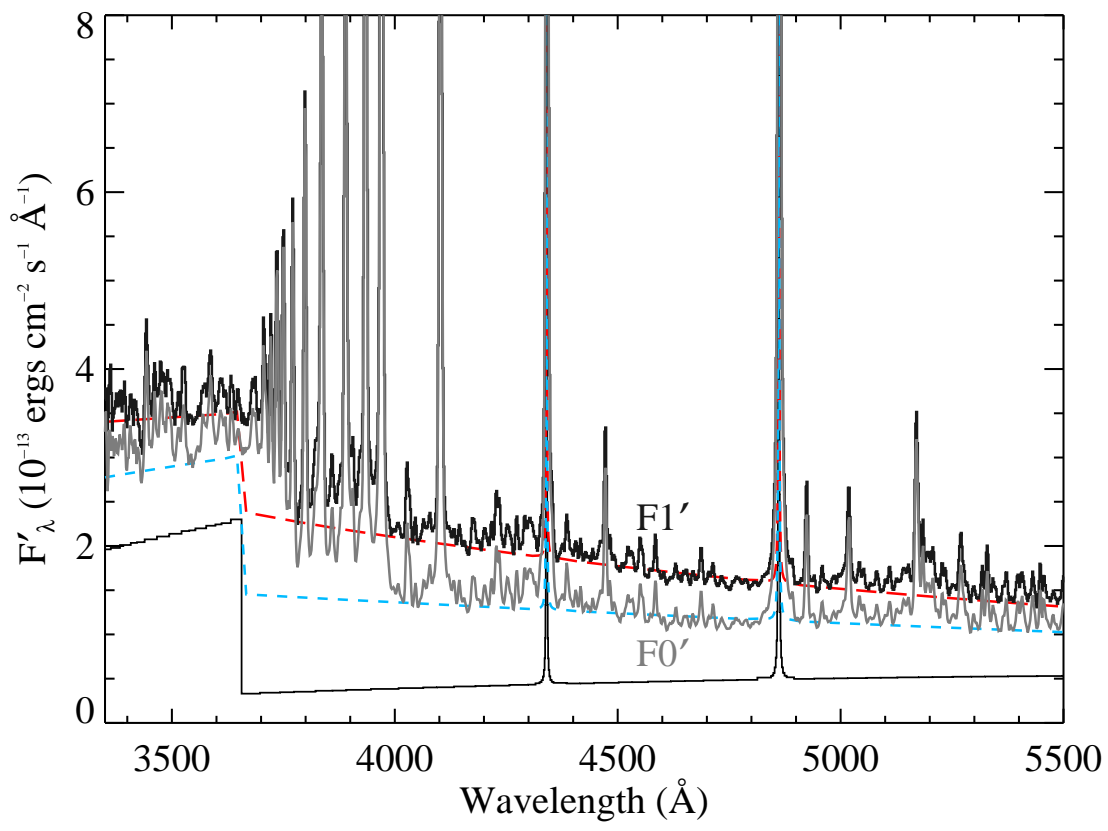

Figure 2. Flare spectra at $t=123.4$ minutes and 126.5 minutes from Figure 1 are shown in grey and black, respectively. The composite model spectra are shown in blue, short dashes $\left(F_{\lambda, \mathrm{RHDF} 11} \times X_{\mathrm{RHDF} 11}+F_{\lambda, \mathrm{HS} 1} \times X_{\mathrm{HS} 1}\right)$ and red, long dashes $\left(F_{\lambda, \mathrm{RHDF} 11} \times X_{\mathrm{RHDF} 11}+F_{\lambda, \mathrm{HS} 1} \times X_{\mathrm{HS} 1}+F_{\lambda, \mathrm{HS} 2} \times X_{\mathrm{HS} 2}\right)$. The RHDF11 spectrum is shown as the thin black line. The continuum veiling effect is apparent from the different heights of the Balmer discontinuity at $\lambda=3646 \AA$.

To model $F 0^{\prime}$, we add the RHDF11 spectrum and a hot spot (HS1) with $T_{\max }=12000 \mathrm{~K}$ (keeping the other parameters the same as described in K10b) with a ratio of filling factors of $10: 1$ and $X_{\mathrm{RHDF} 11}=1.2 \%$, as found in K10a. The total model spectrum is shown as the light blue (short dashes) curve in Figure 2] As in K10b, we model the secondary flare as a hot spot (HS2) with $T_{\max }=20000 \mathrm{~K}$. Adding HS2 to RHDF11 and HS1 gives the red (long dashes) spectrum in Figure 2. The areal coverage of HS2 is 0.46 as large as the areal coverage of HS1. These model spectra match the observed continuum levels at all wavelengths in the figure. Moreover, the continuum veiling / anti-correlation effect is readily apparent: the height of the Balmer jump at $\lambda=3646 \AA$ decreases from black (no hot spots) to blue (one hot spot) to red (two hot spots). The model fluxes are slightly lower than the observed fluxes at $\lambda<3750 \AA$. At these wavelengths, there is a forest of metallic lines (e.g., Fe I, Fe II) that are blended in our low-resolution spectra; our model is satisfactory in matching the underlying level which is likely closer to the actual level of the BaC. Note that in K10a, 
we intentionally used only the BaC from the RHDF11 spectrum to model the continuum, whereas the RHDF11 predictions for the Paschen continuum and photospheric-backwarming components are included in the total fluxes in this work.

The origin of the hot spots is unknown, as they are not predicted by selfconsistent radiative hydrodynamic models that employ a solar-type non-thermal electron heating function (Allred et al., 2006). In the proposed continuum model, we use the fewest number of components necessary to fit the overall shape and reproduce the anti-correlation. However, HS1 may represent a sum of individual decaying hot spots from previously heated flare regions (see below). We are working to produce a grid of phenomenological models which will be used to constrain the column mass of the hot spots, the detailed temperature evolution, and the uniqueness of the continuum fit. Radiative hydrodynamic models (with RADYN) of the gradual phase are also forthcoming and will provide a more accurate representation of the $\mathrm{BaC}$ and photospheric backwarming in the decay phase.

\section{The Solar Analogy}

Figure 3 shows how a spatially resolved observation of the YZ CMi megaflare might have appeared. We use the continuum components and filling factors to make analogies to several flare structures and phenomena observed in large solar flare arcades. The complex morphology of the U-band light curve leads us to speculate that the YZ CMi megaflare involved a large arcade, or several large arcades of flare loops. The main features of Figure 3 are the following:

- $\quad$ BaC (yellow ribbons): Throughout the spectral observations (72 minutes $<t<149$ minutes), the hydrogen $\mathrm{BaC}$ and lines were highly elevated and decreasing (likely from the initial flare peaks in the U-band light curve), implying that this emission had originated from the footpoints of a previously heated magnetic arcade in the flaring chromosphere. These may manifest as a complex of flare ribbons, as is commonly observed in $\mathrm{H} \alpha$ during solar flares (e.g., Rust and Hegwer, 1975; Berlicki et al., 2004; Balasubramaniam et al., 2010). In some solar flares (e.g., Neidig, 1983), the $\mathrm{BaC}$ appears to have a spatial morphology that is more compact than an extended $\mathrm{H} \alpha$ ribbon. Spectroscopic observations are needed to compare the plasma properties and conditions of $\mathrm{BaC}$ and Balmer line emitting ribbons and kernels.

- HS1 (purple spots): Immediately prior to the secondary flare beginning at $t \approx 123$ minutes, a series of phenomenological hot spots (HS1) are present near the photosphere. These hot spots were formed during the previous secondary flares (i.e., at $t \approx 65$ minutes, $\approx 95$ minutes; see K10a), and they are emitting from a total source size that is $\approx 1 / 10$ as large as the area of the chromospheric flare region (e.g., H $\alpha$ ribbons). The spectra of these hot spots have the hydrogen $\mathrm{BaC}$ and lines in absorption. They might be similar to the compact white-light kernels during solar flares, as 
in Wang, Fang, and Ming-DeDing (2007), Fletcher et al. (2007), Jess et al. (2008), or they may be similar to Ellerman-bomb phenomena (see Section 4.1). Also emitting from the photosphere is a larger region heated from chromospheric $(\mathrm{BaC})$ backwarming; we assume the size of this backwarmed region is the same size as the flaring chromosphere.

- HS2 (white spot): The secondary flare at $t \approx 130$ minutes is the result of the formation of a new hot spot (HS2), hotter and smaller than HS1 but at the same column mass. At this time, we see a sudden decline in the $\mathrm{BaC}$ flux. When all of these components are unresolved, as in our stellar spectra, continuum veiling gives rise to the observed anti-correlation. We have placed HS2 assuming it was triggered by a disturbance induced by the huge initial flare peak at $t \approx 28$ minutes. The time-evolution of the $\mathrm{H} \gamma$ and $\mathrm{BaC}$ fluxes in Figure 1d of K10a indicates an apparent lack of new hydrogen Balmer line emitting-regions during the secondary U-band peaks (we cannot definitively determine whether there is a newly-formed hydrogen Balmer-emitting (chromospheric ribbon) component cospatial with the hotspot because the observations are unresolved). Therefore, the disturbance likely propagated through the lower atmosphere, below the height of hydrogen Balmer line formation (upper chromosphere; J. Allred, private communication 2010). Using a range of sound speeds in the lower atmosphere for the speed of the disturbance $\left(\approx 5-10 \mathrm{~km} \mathrm{~s}^{-1}\right)$, we find that HS2 is located at a distance that is approximately $30-60 \mathrm{Mm}\left(\mathrm{R}_{\mathrm{YZ}} \mathrm{CMi} \approx 200 \mathrm{Mm}\right)$ from the site of the initial flare event 1

The composite graphical model is preliminary (see Section 5) and requires comparison to other complex flare events on dMe stars but especially to solar flares where we can spatially resolve each continuum component. Our group is currently working to obtain solar flare data that can be used to test the YZ CMi flare model using DST/ROSA and employing custom continuum filters (Jess et al., 2010b; Kowalski et al., 2011).

\subsection{Are the Secondary Flares Stellar Ellerman Bombs?}

Ellerman bombs are transient, compact phenomena observed near evolving or emerging magnetic fields in solar active regions (Ellerman, 1917; Severny, 1968; Georgoulis et al., 2002, and references therein). A typical signature of Ellerman bombs is emission in the wings and absorption in the core of $\mathrm{H} \alpha$ relative to the nearby plage intensity (e.g., Koval and Severny, 1970; Bruzek, 1972; Fang et al., 2006). The time-evolution properties include mean lifetimes of $\approx$ 10 - 20 minutes and fine-structure variations (Kurokawa et al., 1982; Qiu et al., 2000). In contrast to typical white-light flares, Ellerman bombs have symmetric light curves with similar rise and decay times (Pavne, 1993; Qiu et al., 2000;

\footnotetext{
${ }^{1}$ The white-light and hard X-ray footpoints have been observed to propagate along the polarity inversion line during large solar flares such as the famous 14 July 2000 flare Fletcher and Hudson (2001); Kosovichev and Zharkova (2001); Qiu et al. (2010), but the spatial location of these kernels appears to change much faster, $\approx 170-200 \mathrm{~km} \mathrm{~s}^{-1}$.
} 


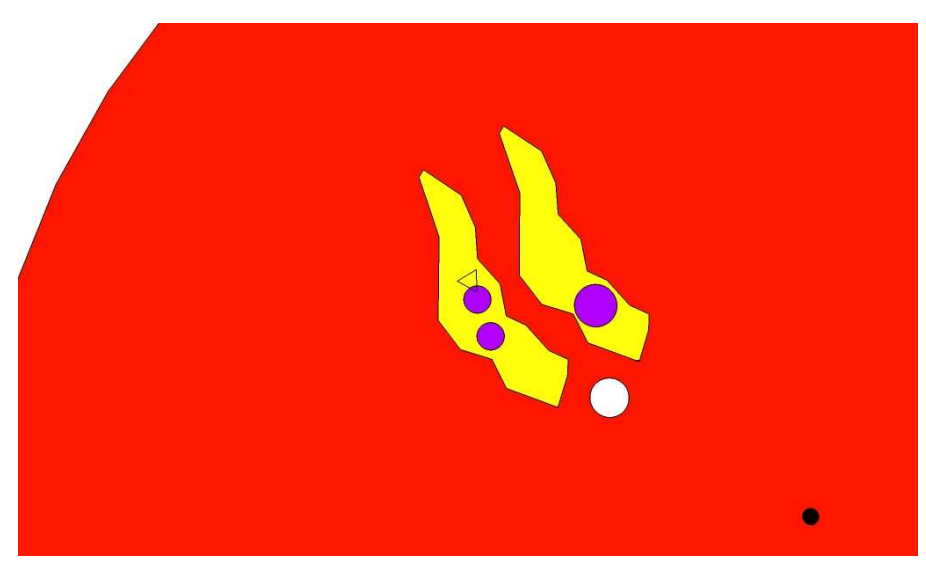

Figure 3. Graphic with continuum components (areas from $t \approx 126.5$ minutes to scale) as they might appear in a spatially resolved observation. The BaC emitting region (yellow) resembles a two-ribbon structure (shown here as symmetric for simplicity) in a thin layer of the heated mid-to-upper chromosphere of previously reconnected magnetic loops. HS1 is shown as a collection of previously formed hot spots (purple) and HS2 is the proposed newly-formed continuum emitting region (white). The triangle indicates the assumed location of the inital flare peak, which generated a disturbance in the lower atmosphere that propagated into the surrounding active region and triggered the hot spots. The black circle helps orient the reader to the center of the star, which has a radius $0.3 R_{\odot}$. The flare region is placed at an arbitrary location on the surface. Several aspects of this cartoon were inspired by observations of solar flare arcades, such as Fletcher and Hudson (2001).

Jess et al., 2010a). The Ellerman-bomb mechanism is not fully understood but has been attributed to magnetic reconnection in the low chromosphere (e.g., Georgoulis et al., 2002).

The secondary flares during the YZ CMi megaflare exhibit several similarities to Ellerman-bomb phenomena on the Sun. Ellerman bombs have also been modeled phenomenologically as temperature bumps at or below the solar temperature minimum region (Fang et al., 2006; Berlicki, Heinzel, and Avrett, 2010). The secondary YZ CMi flares have longer rise times $(\approx$ two-five minutes) and are much more symmetric about the peak compared to other white light flares with similar total energy on YZ CMi $\left(\Delta t_{\text {rise }} \approx 0.5-1.8\right.$ minutes; Moffett, 1974; van den Oord et al., 1996). The absorption features of the blackbodylike continuum component are similar to the line-center absorption observed in $\mathrm{H} \alpha$ and Ca II during Ellerman bombs; unfortunately, our observations do not have sufficient spectral resolution to separate line-center and wing profiles. The preliminary finding (Section 4) that the blackbody-like continuum component does not contain hydrogen-line emission may be consistent with magnetic reconnection taking place in the low atmosphere. However, in contrast to solar Ellerman bombs, which have been observed as a microflare trigger (Jess et al., 2010a), the secondary flares are possibly a consequence of the enormous YZ CMi flare peak event. 


\section{Summary and Future Work}

The time-resolved continuum data obtained during a megaflare on the dM4.5e star YZ CMi demonstrate the power of broad-wavelength coverage, low-resolution spectra, which are unfortunately not currently available in the optical/near-UV for solar flares. In this manuscript, we show that the blackbody-like component (hot star-like emission) of the white-light continuum dominates the spectra during the secondary flares while the $\mathrm{BaC}$ (and Balmer lines) become less important; this observed anti-correlation is explained as continuum veiling. We combine the phenomenological models of the blackbody-like component from K10b and the Allred et al. (2006) RHD model spectrum of the BaC to reproduce the total flare emission at two times during the flare. The filling factors for the decaying $\mathrm{BaC}(+$ backwarming $)$ component, a previously heated hot-spot component, and a newly heated hot spot component are in the ratio of $\approx 10: 1: 0.5$. These areal coverages allow a comparison of each component to be made with observed solarflare structures in large eruptive flare arcades. Although we generally assume that stellar-flare phenomena are simply "scaled-up" versions of solar-flare phenomena, one should not exclude the possibility that stellar flares might have fundamental differences in the white-light continuum, as the energies and timescales of dMe flares can be orders of magnitude larger than solar flares. New solar-flare observations are needed to test the existence of the blackbody-like component and to better understand the properties of the BaC, which could be fully "unveiled" using spatially resolved solar observations.

We provide evidence that the blackbody-like component has several similar properties to solar Ellerman bombs. A few solar flares have been known to exhibit the spectral features (Svestka, 1963) and velocity characteristics (Kosovichev and Zharkova, 2001) of Ellerman bombs. Additional intensity-calibrated continuum measurements of Ellerman bombs and white-light flare kernels on the Sun, such as with DST/ROSA, would help illuminate the differences between these events and provide a comparison to stellar spectra of typical white-light flares and megaflare-size events.

Several aspects of the phenomenological models presented in this work are being improved. In addition to modeling hydrogen with more levels and including metallic transitions and molecular species, a more accurate consideration of charge balance is underway. The correct treatment of charge balance in a modified atmosphere is complicated by non-LTE ionization, but a new version of RH has been provided by $\mathrm{H}$. Uitenbroek to account for this; the authors are currently working on a new suite of hot-spot models.

Acknowledgements This work was first presented at 'The Origin, Evolution, and Diagnosis of Solar Flare Magnetic Fields and Plasmas: Honoring the Contributions of Dick Canfield', a conference that took place from 9-11 August 2010 at HAO/NCAR in Boulder, CO. AFK thanks the organizers of this conference for generous travel assistance and acknowledges support from NSF grant AST 0807205. We gratefully thank H. Uitenbroek for many useful discussions and for our use of the RH code; P. Heinzel, M. Varady, and D. Jess for illuminating conversations about Ellerman bombs; and J. Allred for allowing us to use the detailed output of his RADYN flare models. We also acknowledge Google for our use of its SketchUp application. 
Based on observations obtained with the Apache Point Observatory $3.5 \mathrm{~m}$ Telescope, which is owned and operated by the Astrophysical Research Consortium.

\section{References}

Allred, J.C., Hawley, S.L., Abbett, W.P., Carlsson, M.: 2006, Astrophys. J. 644, 484. doi:10.1086/503314.

Balasubramaniam, K.S., Cliver, E.W., Pevtsov, A., Temmer, M., Henry, T.W., Hudson, H.S., Imada, S., Ling, A.G., Moore, R.L., Muhr, N., Neidig, D.F., Petrie, G.J.D., Veronig, A.M., Vršnak, B., White, S.M.: 2010, Astrophys. J. 723, 587. doi:10.1088/0004-637X/723/1/587.

Berlicki, A., Heinzel, P., Avrett, E.H.: 2010, Mem. Soc. Astronom. Italiana 81, 646.

Berlicki, A., Schmieder, B., Vilmer, N., Aulanier, G., Del Zanna, G.: 2004, Astron. Astrophys. 423, 1119. doi 10.1051/0004-6361:20040259.

Bruzek, A.: 1972, Solar Phys. 26, 94. doi:10.1007/BF00155110.

Carlsson, M., Stein, R.F.: 1994, In: Carlsson, M. (ed.) Chromospheric Dynamics, University, Oslo, 47.

Carlsson, M., Stein, R.F.: 1995, Astrophys. J. Lett. 440, 29. doi 10.1086/187753.

Carlsson, M., Stein, R.F.: 1997, Astrophys. J. 481, 500. doi:10.1086/304043.

Donati-Falchi, A., Smaldone, L.A., Falciani, R.: 1984, Astron. Astrophys. 131, 256.

Eason, E.L.E., Giampapa, M.S., Radick, R.R., Worden, S.P., Hege, E.K.: 1992, Astron. J. 104, 1161. doi:10.1086/116305.

Ellerman, F.: 1917, Astrophys. J. 46, 298. doi:10.1086/142366.

Fang, C., Tang, Y.H., Xu, Z., Ding, M.D., Chen, P.F.: 2006, Astrophys. J. 643, 1325. doi $10.1086 / 501342$

Fletcher, L., Hudson, H.: 2001, Solar Phys. 204, 69. doi: $10.1023 / \mathrm{A}: 1014275821318$.

Fletcher, L., Hannah, I.G., Hudson, H.S., Metcalf, T.R.: 2007, Astrophys. J. 656, 1187. doi $10.1086 / 510446$

Fuhrmeister, B., Liefke, C., Schmitt, J.H.M.M., Reiners, A.: 2008, Astron. Astrophys. 487, 293. doi:10.1051/0004-6361:200809379.

García-Alvarez, D., Jevremović, D., Doyle, J.G., Butler, C.J.: 2002, Astron. Astrophys. 383, 548. doi:10.1051/0004-6361:20011743. 
Georgoulis, M.K., Rust, D.M., Bernasconi, P.N., Schmieder, B.: 2002, Astrophys. J. 575, 506. doi $10.1086 / 341195$

Hartigan, P., Hartmann, L., Kenyon, S., Hewett, R., Stauffer, J.: 1989, Astrophys. J. Supp. Series 70, 899. doi:10.1086/191361.

Hawley, S.L., Pettersen, B.R.: 1991, Astrophys. J. 378, 725. doi: $10.1086 / 170474$.

Hawley, S.L., Allred, J.C., Johns-Krull, C.M., Fisher, G.H., Abbett, W.P., Alekseev, I., Avgoloupis, S.I., Deustua, S.E., Gunn, A., Seiradakis, J.H., Sirk, M.M., Valenti, J.A.: 2003, Astrophys. J. 597, 535. doi:10.1086/378351.

Herczeg, G.J., Hillenbrand, L.A.: 2008, Astrophys. J. 681, 594. doi: $10.1086 / 586728$.

Hessman, F.V., Guenther, E.W.: 1997, Astron. Astrophys. 321, 497.

Hiei, E.: 1982, Solar Phys. 80, 113. doi:10.1007/BF00153427.

Hudson, H.S., Acton, L.W., Hirayama, T., Uchida, Y.: 1992, Pub. Astron. Soc. Japan 44, 77.

Jess, D.B., Mathioudakis, M., Crockett, P.J., Keenan, F.P.: 2008, Astrophys. J. Lett. 688, 119. doi $10.1086 / 595588$.

Jess, D.B., Mathioudakis, M., Browning, P.K., Crockett, P.J., Keenan, F.P.: 2010a, Astrophys. J. Lett. 712, L111. doi:10.1088/2041-8205/712/1/L111.

Jess, D.B., Mathioudakis, M., Christian, D.J., Keenan, F.P., Ryans, R.S.I., Crockett, P.J.: 2010b, Solar Phys. 261, 363. doi:10.1007/s11207-009-9500-0.

Kosovichev, A.G., Zharkova, V.V.: 2001, Astrophys. J. Lett. 550, L105. doi $10.1086 / 319484$.

Koval, A.N., Severny, A.B.: 1970, Solar Phys. 11, 276. doi 10.1007/BF00155226.

Kowalski, A.F., Hawley, S.L., Holtzman, J.A., Wisniewski, J.P., Hilton, E.J.: 2010a, Astrophys. J. Lett. 714, L98. doi $10.1088 / 2041-8205 / 714 / 1 /$ L98

Kowalski, A.F., Hawley, S.L., Holtzman, J.A., Wisniewski, J.P., Hilton, E.J.: 2010b, in Choudhary, D.P., Strassmeier, K.G. (eds.) Physics of Sun and Star Spots, submitted, ArXiv e-prints:1010.0452..

Kowalski, A.F., Mathioudakis, M., Keys, P.H., Hawley, S.L.: 2011, Solar Phys. in preparation.

Kurokawa, H., Kawaguchi, I., Funakoshi, Y., Nakai, Y.: 1982, Solar Phys. 79, 77. doi:10.1007/BF00146974.

Machado, M.E., Rust, D.M.: 1974, Solar Phys. 38, 499. doi $10.1007 /$ BF00155084 
Moffett, T.J.: 1974, Astrophys. J. Supp. Series 29, 1. doi 10.1086/190330.

Neidig, D.F.: 1983, Solar Phys. 85, 285. doi 10.1007/BF00148655.

Neidig, D.F., Kane, S.R.: 1993, Solar Phys. 143, 201. doi 10.1007/BF00619106.

Neidig, D.F., Grosser, H., Hrovat, M.: 1994, Solar Phys. 155, 199. doi:10.1007/BF00670740.

Payne, T.E.W.: 1993, A multiwavelength study of solar Ellerman bombs. PhD thesis, New Mexico State Univ..

Qiu, J., Ding, M.D., Wang, H., Denker, C., Goode, P.R.: 2000, Astrophys. J. Lett. 544, L157. doi $10.1086 / 317310$.

Qiu, J., Liu, W., Hill, N., Kazachenko, M.: 2010, Astrophys. J. 725, 319. doi $10.1088 / 0004-637 X / 725 / 1 / 319$.

Rust, D.M., Hegwer, F.: 1975, Solar Phys. 40, 141. doi:10.1007/BF00183158.

Severny, A.B.: 1968, In: Oehman, Y. (ed.) Mass Motions in Solar Flares and Related Phenomena, Almqvist and Wiksell, Stockholm, 71.

Svestka, Z.: 1963, Bull. Astronom. Instit. Czech. 14, 234.

Uitenbroek, H.: 2001, Astrophys. J. 557, 389. doi 10.1086/321659

van den Oord, G.H.J., Doyle, J.G., Rodono, M., Gary, D.E., Henry, G.W., Byrne, P.B., Linsky, J.L., Haisch, B.M., Pagano, I., Leto, G.: 1996, Astron. Astrophys. 310, 908.

Wang, L., Fang, C., Ming-DeDing: 2007, Chin. J. Astron. Astrophys. 7, 721. doi: $10.1088 / 1009-9271 / 7 / 5 / 13$

Zhilyaev, B.E., Romanyuk, Y.O., Svyatogorov, O.A., Verlyuk, I.A., Kaminsky, B., Andreev, M., Sergeev, A.V., Gershberg, R.E., Lovkaya, M.N., Avgoloupis, S.J., Seiradakis, J.H., Contadakis, M.E., Antov, A.P., KonstantinovaAntova, R.K., Bogdanovski, R.: 2007, Astron. Astrophys. 465, 235. doi:10.1051/0004-6361:20065632 